Article

\title{
A Lévy-Driven Stochastic Queueing System with Server Breakdowns and Vacations
}

\author{
Yi Peng ${ }^{1}$ and Jinbiao $\mathrm{Wu}^{2, * \mathbb{D}}$ \\ 1 School of Mathematical Science, Changsha Normal University, Changsha 410100, China; \\ mailto:pengyiqueue@163.com \\ 2 School of Mathematics and Statistics, Central South University, Changsha 410083, China \\ * Correspondence: wujinbiao@csu.edu.cn
}

Received: 24 June 2020; Accepted: 28 July 2020; Published: 29 July 2020

\begin{abstract}
Motivated by modelling the data transmission in computer communication networks, we study a Lévy-driven stochastic fluid queueing system where the server may subject to breakdowns and repairs. In addition, the server will leave for a vacation each time when the system is empty. We cast the workload process as a Lévy process modified to have random jumps at two classes of stopping times. By using the properties of Lévy processes and Kella-Whitt martingale method, we derive the limiting distribution of the workload process. Moreover, we investigate the busy period and the correlation structure. Finally, we prove that the stochastic decomposition properties also hold for fluid queues with Lévy input.
\end{abstract}

Keywords: fluid queueing systems; Lévy processes; martingales; queues with Lévy input; server breakdowns and vacations; stochastic decomposition

\section{Introduction}

Lévy processes are very important stochastic processes with stationary and independent increments. They are one of the most important families of stochastic processes arising in many areas of probability theory and stochastic operations research. They cover a number of very important and well-studied stochastic processes as special cases, such as Brownian motion, compound Poisson processes, stable processes and subordinators. Lévy processes are widely used as models in the study of stochastic service systems, insurance risks, stochastic inventory systems, reliability engineering, computer communication systems, stochastic finance systems and so on. For a review of main results and methods of Lévy processes and their applications, the reader may refer to the excellent books by Bertoin [1], Sato [2], Applebaum [3], and Kyprianou [4].

Recently, queues with server breakdowns and vacations have got a lot of attention due to their wide and interesting applications especially in computer communication networks and manufacturing systems. Gray et al. [5] analyzed a multiple-vacation $M / M / 1$ queueing model, where the service station is subject to breakdown while in operation. Ke [6] studied the control policy in $M^{[X]} / M / 1$ queue with server breakdowns and multiple vacations. Jain and Jain [7] dealt with a single server working vacation queueing model with multiple types of server breakdowns. Wu and Yin [8] gave a detailed analysis on an $M / G / 1$ retrial queue with non-exhaustive random vacations and unreliable server. Yang and $\mathrm{Wu}[9]$ investigated the $\mathrm{N}$-policy $M / M / 1$ queueing system with working vacation and server breakdowns. An excellent and comprehensive study on the queueing systems with vacations, including some applications such as traffic systems, logistic systems, production/inventory systems, communication systems, and computer systems, can be found in the book by Tian and Zhang [10]. However, most of the papers are focused on queues where customers arrive at the system according to independent Poisson processes or geometrical arrival processes. Queueing systems with Lévy input (or Lévy-driven queues) are still not well investigated in literature. Queuing systems 
with Lévy input have been attracting increasing attention in the applied probability and stochastic operations research communities. For one thing, Lévy-driven queues cover the classical $M / G / 1$ queue and the reflected Brownian motion as special cases. For another thing, Lévy-driven fluid queues are more suitable to model data transmission in computer communication network. Since the arrival data is very huge in big data era, it is more appropriate to describe the arrival of data by fluids. Kella and Whitt [11] first considered Lévy processes with secondary jump input which were applied to analyze queues with server vacations. Lieshout and Mandjes [12] deduced tail asymptotics of a two-node tandem queue with spectrally positive Lévy input. Boxma and Kella [13] analyzed a generic class of Lévy-driven queuing systems with server vacation. Dębicki and Mandjes [14] provided a survey on Lévy-driven queues. Dębicki et al. [15] focused on transient analysis of Lévy-driven tandem queues. Palmowski et al. [16] considered a controlled fluid queuing model with Lévy input. Boxma and Kella [17] generalized known workload decomposition results for Lévy queues with secondary jump inputs and queues with server vacations or service interruptions. Recently, Ivanovs and Mandjes [18] presented a detailed analysis of various random quantities related to the transient of a Lévy-driven queue. Koops et al. [19] studied the stationary workload distribution of a fluid tandem queue with Lévy input in heavy traffic. Debicki et al. [20] derived exact large buffer asymptotics for a two-class generalized processor sharing system with Lévy input. Dai [21] introduced a unified stochastic queueing network of coupled forward-backward stochastic differential equations with Lévy jumps and double completely-S skew reflections. Ravner et al. [22] gave semi-parametrically estimating the input process to a Lévy-driven queue by sampling the workload process at Poisson times. Berkelmansa et al. [23] dealt with the workload correlation function of a queue with general Lévy input, i.e., queue fed by a spectrally two-sided Lévy process. However, to the best of our knowledge, no work on Lévy queues with server breakdowns and vacations is found in the queueing literature.

In this paper, we consider a single-server Lévy-driven fluid queue with multiple vacations (exhaustive service) and a server subject to breakdowns and repairs. A breakdown at the server is represented by the arrival of a failure. The principal purpose of the present paper is to apply the martingale results which were derived in Kella and Whitt [24] to investigate the stochastic dynamics of the system and realize an extensive analysis of the system from the transient virtual waiting time process to the steady-state distribution of the waiting time process. In addition, we give a detailed analysis of the system busy period and the queue's correlation structure. Furthermore, we establish stochastic decomposition principles for the system. Our results extend and unify previous results for $M / G / 1$ queue with server breakdowns and vacations and Lévy-driven queues with server vacations.

Let us briefly describe the contents of this paper. In Section 2, we introduce a few basic knowledge about Lévy processes and martingales. In Section 3, we give the model description and formulate the model as a Lévy process modified to have random jumps at two classes of stopping times. In Section 4 , we characterize the steady-state distribution of the virtual waiting time process and the mean length of the busy period. In Section 5, we address the transient distribution of the waiting time process as well as the queue's correlation structure. In Section 6, we present two stochastic decomposition results. Finally, a summary of the results is presented in Conclusion.

\section{Preliminaries on Lévy Processes}

In this section, we mainly present several notations, definitions and propositions concerning Lévy processes. For more details, see [1-4].

Write $\mathbb{R}=(-\infty, \infty)$ and $\mathbb{R}_{+}=[0, \infty)$. Let $\left(\Omega, \mathcal{F}, \mathbb{P},\left\{\mathcal{F}_{t \geq 0}\right\}\right)$ be a complete probability space equipped with a filtration $\mathcal{F}_{t \geq 0}$, i.e., an increasing family of sub-fields, which satisfies the usual conditions. That is, each $\mathcal{F}_{t}$ is $\mathbb{P}$-complete and $\mathcal{F}_{t}=\cap_{s}>t \mathcal{F}_{s}$ for every $t$. Throughout the paper, adapted, stopping times and martingales will be defined with respect to this filtration. In what follows, given a càdlàg (right continuous left limit) function of finite variation $f$, denote $\Delta f(t)=f(t)-f(t-)$ the jump of $f$ at $t$, where $f(t-)=\lim _{s \uparrow t} f(s)$, and $f^{d}(t)=\sum_{0 \leq s \leq t} \Delta f(s)$ and $f^{c}(t)=f(t)-f^{d}(t)$. 
Definition 1. Let $X=\left(X_{t}, t \geq 0\right)$ be a stochastic process defined on a probability space $(\Omega, \mathcal{F}, \mathbb{P})$. $X$ is called a Lévy process if

1. $X_{0}=0$ (a.s.);

2. X has stationary and independent increments;

3. $X$ is continuous in probability, i.e., for all $a>0$ and for all $s \geq 0$,

$$
\lim _{t \rightarrow s} \mathbb{P}\left(\left|X_{t}-X_{s}\right|>a\right)=0 .
$$

A key result on Lévy processes is the Lévy-Khintchine formula given by the following proposition and it is the cornerstone for our analysis. Let $v$ be a Borel measure defined on $\mathbb{R}-\{0\} . v$ is called a Lévy measure if $\int_{\mathbb{R}-\{0\}}\left(|x|^{2} \wedge 1\right) v(d x)<\infty$.

Proposition 1. Let X be a Léry process with Léry measure v. Then

$$
\mathbb{E} e^{i \theta X_{t}}=e^{t \psi(\theta)}, \quad t \geq 0,
$$

where

$$
\psi(\theta)=i \mu \theta-\frac{1}{2} \theta^{2} \sigma^{2}+\int_{\mathbb{R}-\{0\}}\left(e^{i \theta x}-1-i \theta x \mathbf{1}_{|x|<1}\right) v(d x),
$$

$\mu \in \mathbb{R}$ and $\sigma \geq 0$. Conversely, given $v, \mu, \sigma^{2}$, there exists a Lévy process which is unique in distribution. Moreover, the jump process of $X$ is a Poisson point process with characteristic measure $v$, that is

$$
v(\Lambda)=\mathbb{E}\left[\sum_{0<s \leq 1} \mathbf{1}_{\Lambda}\left(\Delta X_{s}\right)\right] .
$$

We call the function $\psi(\cdot)$ the Lévy exponent of the Lévy process $X$. It is well known that there always exists a version with sample paths of $X$ that are càdlàg. Hence it is a strong Markov process. In view of this result, throughout this paper, we assume that all Lévy processes mentioned are càdlàg. If $v(-\infty, 0)=0$, then the Laplace-Stieltjes transform exists and is given by $\mathbb{E} \exp \left(-\theta X_{t}\right)=\exp (t \varphi(\theta))$ where

$$
\varphi(\theta)=\log \mathbb{E} \exp \left\{-\theta X_{1}\right\}=-\mu \theta+\frac{1}{2} \theta^{2} \sigma^{2}+\int_{\mathbb{R}_{+}}\left(e^{-\theta x}-1+\theta x \mathbf{1}_{|x|<1}\right) v(d x),
$$

here the function $\varphi(\cdot)$ is called the Laplace-Stieltjes exponent of the Lévy process. In this paper, we focus only on the spectrally positive Lévy process, i.e., $v(-\infty, 0)=0$. Generally, in queueing theory, there are many stochastic processes associated with spectrally positive Lévy processes. For example, the workload process of the M/G/1 queue is a reflected spectrally positive Lévy process. However, in risk theory, the risk processes are usually modelled by spectrally negative Lévy processes, i.e., $v(0, \infty)=0$. The reader can refer to the excellent book by Kyprianou [4] for a review of main results and methods about the spectrally positive Lévy processes and spectrally negative Lévy processes and their applications in queueing theory and risk theory. For any spectrally positive Lévy process $X$ with $E X_{1}<0$. Denote by $T^{\xi}=\inf \left\{t \mid X_{t}=-\xi\right\}$ for any non-negative random variable $\xi$ which is independent of $X$. Then for $\beta>0$, it follows by Theorem 3.12 in [4] that

$$
E \exp \left\{-\beta T^{\tilde{\xi}}\right\}=\exp \left\{-\varphi^{-1}(\beta) \xi\right\}, \quad E T^{\tilde{\xi}}=\frac{E \xi}{-E X_{1}} .
$$


We now give the generalized Pollaczek-Khinchine formula associated with a reflected Lévy process with no negative jumps which is also a celebrated formula in queueing theory. Given some random variable $0 \leq R_{0} \in \mathcal{F}_{0}$, let

$$
I_{t}=\left(-\inf _{0 \leq s \leq t} X_{s}-R_{0}\right)^{+}, \quad R_{t}=R_{0}+X_{t}+I_{t}, \quad t \geq 0 .
$$

Then $R$ is a reflected Lévy process with $I$ being its local time at zero. Since $X$ has no negative jumps, $I$ is continuous with $I_{0}=0$ and is the minimal right-continuous nondecreasing process such that $R_{t} \geq 0$ for all $t$. The process $\left\{I_{t}, t \geq 0\right\}$ must only increase when $R_{t}=0$, so that

$$
\int_{0}^{\infty} \mathbf{1}_{\left\{R_{t}>0\right\}} d I_{t}=0
$$

The following result is the famous (generalized) Pollaczek-Khinchine formula in queueing theory. The reader can refer to the papers [11] and [14] for its proof.

Proposition 2. If $X$ is a spectrally positive Lévy process such that $E X_{1}<0$, then

$$
\lim _{t \rightarrow \infty} \mathbb{E} e^{-\theta R_{t}}=\frac{\theta \varphi^{\prime}(0)}{\varphi(\theta)}, \quad \theta>0 .
$$

We end this section by presenting the famous Kella-Whitt martingale associated with a spectrally positive Lévy process $X$ with exponent $\varphi(\theta)$ that will be very useful for the analysis the stationary distribution of the workload process of our fluid queue. The Kella-Whitt martingale takes the name from Kella and Whitt [24] and is showed in the following proposition.

Proposition 3. Let $X$ be a spectrally positive Lévy process with exponent $\varphi(\theta)$. Let $L_{t}=\int_{0}^{t} d L_{s}^{c}+\sum_{0 \leq s \leq t} \Delta L_{s}$ be an adapted càdlàg process of finite variation with continuous part $\left\{L_{t}^{c}\right\}$ and jumps $\Delta L_{s}=L_{s}-L_{s-}$. Define $Z_{t}=Z_{0}+X_{t}+L_{t}$, where $Z_{0}$ is measurable with respect to $\mathcal{F}_{0}$. Then

$$
\begin{aligned}
M_{t}= & \varphi(\theta) \int_{0}^{t} \exp \left\{-\theta Z_{s}\right\} d s+\exp \left\{-\theta Z_{0}\right\}-\exp \left\{-\theta Z_{t}\right\}-\theta \int_{0}^{t} \exp \left\{-\theta Z_{s}\right\} d Y_{s}^{c} \\
& +\sum_{0 \leq s \leq t} \exp \left\{-\theta Z_{s}\right\}\left[1-\exp \left\{\theta \Delta L_{s}\right\}\right]
\end{aligned}
$$

is a local martingale, where the integral $\int_{0}^{t} \exp \left\{-\theta Z_{s}\right\} d Y_{s}^{c}$ is the Stieltjes integral. In addition, if the expected variation of $\left\{L_{t}^{c}, t \geq 0\right\}$ and the expected number of jumps of $\left\{L_{t}, t \geq 0\right\}$ are finite on every finite interval and $\left\{Z_{t}, t \geq 0\right\}$ is a non-negative process (or bounded below), then $\left\{M_{t}, t \geq 0\right\}$ is a martingale.

The Kella-Whitt martingale is a stochastic integral with respect to the Wald martingale $N_{t}=\exp \left\{-\theta X_{t}-\varphi(\theta) t\right\}$ and the reader may refer to the paper [24] or the book [4] for its proof.

\section{Model Formulation}

We consider a single fluid queue with a (nondecreasing) Lévy input. For any $t \geq 0$, let $X_{t}$ be the cumulative input of fluid over the interval $[0, t]$. We assume that $X$ is a one-dimensional right continuous subordinator, that is, a nondecreasing Lévy process, having the exponent

$$
\phi(\theta)=-a \theta+\int_{\mathbb{R}_{+}}\left(e^{-\theta x}-1\right) v(d x),
$$

where $a \geq 0$ and $\int_{\mathbb{R}_{+}} x v(d x)<\infty$. Let $S(t)$ be the cumulative available processing over the interval $[0, t]$. Here, we assume the server processes the fluid at a constant deterministic rate $r$ (whenever the fluid level is positive). So that $S(t)=r t, t \geq 0$. We assume the storage space is unlimited. 
Let $\left\{\tau_{n}, n \geq 1\right\}$ be the strictly increasing sequence of stopping times at which the server fails. When the server fails, it is repaired immediately and the time required to repair it is a positive random variable $\xi_{n}$ which is $\mathcal{F}_{\tau_{n}}$-measurable for $n \geq 1$. That is,

$$
\tau_{0}=0, \xi_{0}=0, \tau_{n}=\inf \left\{t \mid t>\tau_{n-1}+\xi_{n-1}, \text { the server is broken at time } t\right\}, n=1,2,3, \cdots .
$$

When the system becomes empty, the server takes a vacation of random length $\eta_{n}$ which is $\mathcal{F}_{\sigma_{n}}$-measurable for $n \geq 1$, where $\left\{\sigma_{n}, n \geq 1\right\}$ is a strictly increasing sequence of stopping times at which the server is leaving for a vacation. Vacations continue until, one returns from a vacation, the server finds the system is non-empty.

Let $W_{t}$ represent the workload level at time $t$ (the virtual-waiting-time process). Denote the initial workload by $W_{0}$ which is measurable with respect to $\mathcal{F}_{0}$. Then $W_{t}$ can be defined by (the empty sum is zero)

$$
W_{t}=W_{0}+X_{t}-r t+\sum_{i=1}^{N_{t}^{R}} \xi_{i}+\sum_{i=1}^{N_{t}^{V}} \eta_{i}, \quad t \geq 0
$$

where

$$
\begin{gathered}
N_{t}^{R}=\sup \left\{n \mid \tau_{n} \leq t\right\}, \quad N_{t}^{V}=\sup \left\{n \mid \sigma_{n} \leq t\right\}, \\
\sigma_{n}=\inf \left\{t \geq 0 \mid W_{0}+X_{t}-r t+\sum_{i=1}^{N_{t}^{R}} \xi_{i}+\sum_{i=1}^{n-1} \eta_{i}=0\right\}, \quad n \geq 1 .
\end{gathered}
$$

The reader can see the Figure 1 for a diagram of a sample path of $W_{t}$ of this model. Define $Y_{t}=X_{t}-r t$. Then $Y$ is a spectrally positive Lévy process with exponent $\varphi(\theta)=\phi(\theta)+r \theta$. Denote $\rho=-\phi^{\prime}(0)$ and impose the conditions $\rho<r$ and $\mathbb{E}\left|Y_{t}\right|<\infty$ for all $t \geq 0$.

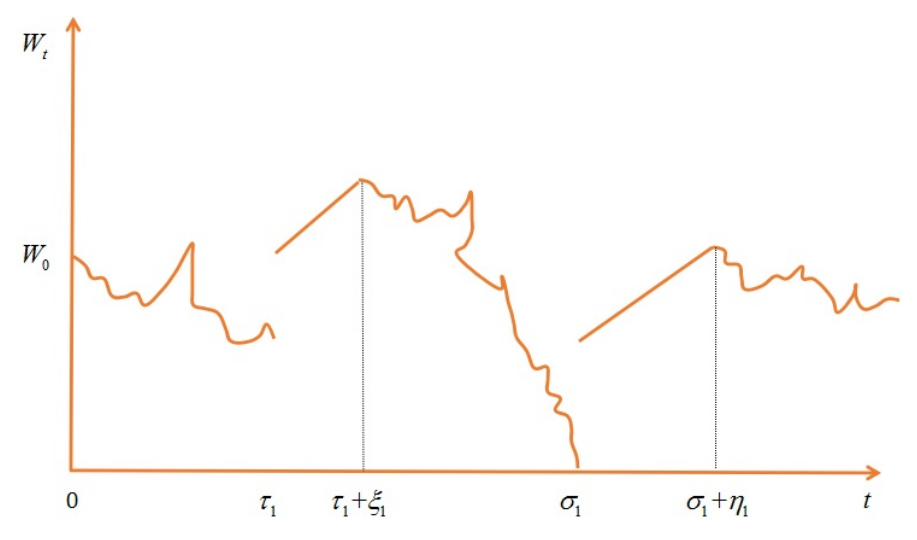

Figure 1. A diagram of a sample path of $W_{t}$.

\section{The Steady-State Distribution}

In this section, we characterize the limiting distribution of the workload $W_{t}$. Denote by $\stackrel{d}{\rightarrow}$ the convergence in distribution and $\stackrel{p}{\rightarrow}$ the convergence in probability. Under the condition $\rho<r$, we suppose $W_{t} \stackrel{d}{\rightarrow} W$ and $t^{-1} \mathbb{E} W_{t} \rightarrow 0$, as $t \rightarrow \infty$, where $W$ is a random variable.

In order to derive the steady-state distribution of the virtual waiting time $W_{t}$, we give the following lemmas. 
Lemma 1. If $E\left(N_{t}^{R}+N_{t}^{V}\right)<\infty$ for all $t$, then $\left\{M_{t}, t \geq 0\right\}$ is a zero-mean real-valued martingale with respect to $\left\{\mathcal{F}_{t}, t \geq 0\right\}$, where

$$
M_{t}=\varphi(\theta) \int_{0}^{t} e^{-\theta W_{s}} d s+e^{-\theta W_{0}}-e^{-\theta W_{t}}-\sum_{k=1}^{N_{t}^{R}}\left[e^{-\theta\left(W_{\tau_{k}}-\tau_{k}\right)}-e^{-\theta W_{\tau_{k}}}\right]-\sum_{k=1}^{N_{t}^{V}}\left(1-e^{-\theta \eta_{k}}\right) .
$$

Proof of Lemma 1. Let $H_{t}=\sum_{i=1}^{N_{t}^{R}} \xi_{i}+\sum_{i=1}^{N_{t}^{V}} \eta_{i}$. Then $W_{t}=W_{0}+Y_{t}+H_{t}$ and $\left\{H_{t}, t \geq 0\right\}$ is an adapted process with finite variation, and $H_{t}^{c}=0$ for all $t \geq 0$. Since $E\left(N_{t}^{R}+N_{t}^{V}\right)<\infty$, by Proposition 3 and through a trivial manipulation, we get that (2) is a martingale.

Lemma 2. (Wald's lemma) Let $T$ be a nonnegative random variable with possible integer values $0,1,2, \cdots$ and $\mathbb{E} T<\infty$. If $\left\{\zeta_{n}, n \geq 1\right\}$ is an i.i.d. sequence with $\zeta_{n}$ independent of $\mathbf{1}_{\{T \geq n\}}$ and $\mathbb{E} \zeta_{1}<\infty$, then

$$
\mathbb{E} \sum_{n=1}^{T} \zeta_{n}=\left(\mathbb{E} \zeta_{1}\right)(\mathbb{E} T)
$$

With the help of the above two lemmas, we can derive the limiting distribution of the workload process provided in the following theorem which is the most important result of this paper.

Theorem 1. Let $\left\{\xi_{n}, n \geq 1\right\}$ and $\left\{\eta_{n}, n \geq 1\right\}$ be two positive i.i.d. sequences with $\mathbb{E} \xi_{1}<\infty$ and $\mathbb{E} \eta_{1}<\infty$, respectively. Suppose that $n^{-1} \tau_{n} \stackrel{p}{\rightarrow} \lambda_{R}^{-1}$ and $n^{-1} \sigma_{n} \stackrel{p}{\rightarrow} \lambda_{V}^{-1}$ as $n \rightarrow \infty$ for $0<\lambda_{R}^{-1}<\infty$ and $0<\lambda_{V}^{-1}<\infty$, respectively. Further, assume that $\left\{W_{\tau_{k}}, k \geq 1\right\}$ and $\left\{W_{\tau_{k}}-\xi_{k}, k \geq 1\right\}$ are stationary and ergodic that $\left(W_{\tau_{k}}-\xi_{k}, W_{\tau_{k}}\right) \stackrel{d}{\rightarrow}\left(W^{-}, W^{+}\right)$where $W^{-}, W^{+}$are two proper random variables. If $\xi_{n}$ is independent of $\mathbf{1}_{\left\{N_{1}^{R} \geq n\right\}}$ and $\eta_{n}$ is independent of $\mathbf{1}_{\left\{N_{1}^{V} \geq n\right\}}$, then for $\theta>0$,

$$
\begin{gathered}
\lambda_{R}=\frac{p \varphi^{\prime}(0)}{\mathbb{E} \xi_{1}}, \quad \lambda_{V}=\frac{(1-p) \varphi^{\prime}(0)}{\mathbb{E} \eta_{1}}, \\
\lim _{t \rightarrow \infty} t^{-1} \mathbb{E} \sum_{k=1}^{N_{t}^{R}} \xi_{k}=-p \mathbb{E} Y_{1}, \quad \lim _{t \rightarrow \infty} t^{-1} \mathbb{E} \sum_{k=1}^{N_{t}^{V}} \eta_{k}=-(1-p) \mathbb{E} Y_{1}, \\
\lim _{t \rightarrow \infty} \mathbb{E} e^{-\theta W_{t}}=\mathbb{E} e^{-\theta W}=\frac{\theta \varphi^{\prime}(0)}{\varphi(\theta)}\left[p \frac{\mathbb{E} e^{-\theta W^{-}}-\mathbb{E} e^{-\theta W^{+}}}{\theta \mathbb{E} \xi_{1}}+(1-p) \frac{1-\mathbb{E} e^{-\theta \eta_{1}}}{\theta \mathbb{E} \eta_{1}}\right],
\end{gathered}
$$

where $0 \leq p \leq 1$.

Proof of Theorem 1. Dividing (1) by $t$ and taking expectation, we arrive at

$$
t^{-1} \mathbb{E} W_{t}=t^{-1} \mathbb{E} W_{0}+\mathbb{E} Y_{1}+t^{-1} \mathbb{E} \sum_{k=1}^{N_{t}^{R}} \xi_{k}+t^{-1} \mathbb{E} \sum_{k=1}^{N_{t}^{V}} \eta_{k}
$$

where $t^{-1} \mathbb{E} W_{t} \rightarrow 0$ by the assumption. Thus, we have proved (4).

Taking $W_{0}=W^{*}$ where $W^{*}$ is an independent copy of $W,\left\{W_{t}, t \geq 0\right\}$ becomes stationary. By Lemma 2 and (1), we get

$$
\mathbb{E} N_{1}^{R} \mathbb{E} \xi_{1}+\mathbb{E} N_{1}^{V} \mathbb{E} \eta_{1}=-\mathbb{E} \Upsilon_{1} .
$$


So that

$$
\mathbb{E} N_{1}^{R} \mathbb{E} \xi_{1}=-p \mathbb{E} Y_{1}, \quad \mathbb{E} N_{1}^{V} \mathbb{E} \eta_{1}=-(1-p) \mathbb{E} Y_{1}
$$

Hence

$$
\mathbb{E} N_{1}^{R}=\frac{p \varphi^{\prime}(0)}{\mathbb{E} \xi_{1}}, \quad \mathbb{E} N_{1}^{V}=\frac{(1-p) \varphi^{\prime}(0)}{\mathbb{E} \eta_{1}}
$$

Conditions $n^{-1} \tau_{n} \stackrel{p}{\rightarrow} \lambda_{R}^{-1}$ and $n^{-1} \sigma_{n} \stackrel{p}{\rightarrow} \lambda_{V}^{-1}$ as $n \rightarrow \infty$ imply that $t^{-1} N_{t}^{R} \stackrel{p}{\rightarrow} \lambda_{R}$ and $t^{-1} N_{t}^{V} \stackrel{p}{\rightarrow} \lambda_{V}$. Together with $t^{-1} N_{t}^{R} \rightarrow \mathbb{E} N_{1}^{R}$ and $t^{-1} N_{t}^{V} \rightarrow \mathbb{E} N_{1}^{V}$ with probability (w.p.) 1 as $t \rightarrow \infty$, this implies (3).

From Lemma 2, optional stopping at $t=1$ in (2) yields

$$
0=\varphi(\theta) \mathbb{E} e^{-\theta W}-\mathbb{E} N_{1}^{R}\left[\mathbb{E} e^{-\theta W^{-}}-\mathbb{E} e^{-\theta W^{+}}\right]-\mathbb{E} N_{1}^{V}\left(1-\mathbb{E} e^{-\theta \eta_{1}}\right)
$$

Substituting (8) into (9), we obtain (5).

Remark 1. From [25], we have $\varphi(\theta)=0$ for some $\theta>0$ if and only if $Y_{t}$ has a lattice distribution. However, for a Lévy process with no negative jumps and $\mathbb{E} Y_{t}<0$, it is not possible. In addition, when $N_{t}^{R} \equiv 0$ for all $t$, i.e., $p=0$, the present system reduces to the Lévy process-driven queue with server vacations. It may be noted that the Equation (5) after putting $p=0$ agrees with the Equation (4.6) presented in Kella and Whitt [11]. We should note that when $N_{t}^{V} \equiv 0$ for all $t$, the present system reduces to the Léry process-driven queue with server breakdowns. However, the Equation (5) in Theorem 1 after putting $p=1$ cannot characterize the limiting distribution of Lévy-driven queue with server breakdowns. This is because when $p=1$, the reduced process $\left\{W_{t}, t \geq 0\right\}$ is not a non-negative process.

Corollary 1. When the system is stable, we have

$$
\begin{gathered}
\omega=\mathbb{E} W=\frac{\varphi^{\prime \prime}(0)}{2 \varphi^{\prime}(0)}+p \frac{\mathbb{E}\left(W^{+}\right)^{2}-\mathbb{E}\left(W^{-}\right)^{2}}{2 \mathbb{E} \xi_{1}}+(1-p) \frac{\mathbb{E} \eta_{1}^{2}}{2 \mathbb{E} \eta_{1}}, \\
v=\mathbb{V a r} W=\frac{1}{3} \frac{\varphi^{\prime \prime \prime}(0)}{\varphi^{\prime}(0)}-\frac{1}{4}\left(\frac{\varphi^{\prime \prime}(0)}{\varphi^{\prime}(0)}\right)^{2}+(1-p)\left[\frac{1}{3} \frac{\mathbb{E} \eta_{1}^{3}}{\mathbb{E} \eta_{1}}-\frac{1}{4}\left(\frac{\mathbb{E} \eta_{1}^{2}}{\mathbb{E} \eta_{1}}\right)^{2}\right] \\
+p\left[\frac{1}{3} \frac{\mathbb{E}\left(W^{+}\right)^{3}-\mathbb{E}\left(W^{-}\right)^{3}}{\mathbb{E} \xi_{1}}-\frac{1}{4}\left(\frac{\mathbb{E}\left(W^{+}\right)^{2}-\mathbb{E}\left(W^{-}\right)^{2}}{\mathbb{E} \xi_{1}}\right)^{2}\right] .
\end{gathered}
$$

Next, we analyze the system busy period which is defined as the time period which starts at the epoch when a vacation is completed and the server begins processing the fluid and ends at the next epoch when the system is empty. Note that a busy period includes the normal processing time of the fluid in the system and some possible repair times of the server due to the server breakdowns.

Lemma 3. Define $T_{n}=\inf \left\{t \mid Y_{t}+\sum_{k=1}^{N_{t}^{R}} \xi_{k}+\sum_{i=1}^{n} B_{i}=0\right\}, n \geq 1$, where $\left\{B_{i}, i \geq 1\right\}$ is a positive i.i.d. sequence with $\mathbb{E} B_{1}<\infty$. If $0 \leq p<1$, then

$$
\mathbb{E} T_{n}=\frac{n \mathbb{E} B_{1}}{(1-p) \varphi^{\prime}(0)} .
$$

Proof of Lemma 3. Let $T_{n}^{b}=\inf \left\{t \mid Y_{t}+\sum_{k=1}^{N_{t}^{R}} \xi_{k}+n b=0\right\}, b>0, n \geq 1$. Since $Y$ is a spectrally positive Lévy process, $\left\{T_{n}^{b}, n \geq 1\right\}$ is a random walk with $0<T_{n}^{b}<T_{n+1}^{b}$ w.p. 1 . Thus, $\mathbb{E} T_{n}^{b}=n \mathbb{E} T_{1}^{b}$. Since $\mathbb{E} Y_{t}<0$ and $0 \leq p<1, \mathbb{E}\left(Y_{t}+\sum_{k=1}^{N_{t}^{R}} \xi_{k}\right)=-(1-p) \varphi^{\prime}(0) t<0$. By Theorem 8.4.4 in [25], 
we have $\mathbb{E} T_{1}^{b}<\infty$. Since $\mathbb{E}\left|Y_{t}\right|<\infty, t^{-1} Y_{t} \rightarrow \mathbb{E} Y_{1}$ w.p.1 as $t \rightarrow \infty$ by the Kolmogorov strong law of large numbers. Taking into account the fact that $Y_{T_{n}^{b}}+\sum_{k=1}^{N_{T_{n}^{b}}^{R}} \xi_{k}=-n b$, we get

$$
\begin{aligned}
-b & =n^{-1}\left(Y_{T_{n}^{b}}+\sum_{k=1}^{N_{T_{n}^{b}}^{R}} \xi_{k}\right)=n^{-1} T_{n}^{b}\left[\left(T_{n}^{b}\right)^{-1} Y_{T_{n}^{b}}+\left(T_{n}^{b}\right)^{-1} \sum_{k=1}^{N_{T_{n}^{b}}^{R}} \xi_{k}\right] \\
& \longrightarrow \mathbb{E} T_{1}^{b}\left[\mathbb{E} Y_{1}+\left(\mathbb{E} N_{1}^{R}\right)\left(\mathbb{E} \xi_{1}\right)\right]=\mathbb{E} T_{1}^{b}\left[-(1-p) \varphi^{\prime}(0)\right] \text { w.p. } 1 \text { as } n \rightarrow \infty .
\end{aligned}
$$

So that $\mathbb{E} T_{n}^{b}=\frac{n b}{(1-p) \varphi^{\prime}(0)}$. Finally, by conditioning and unconditioning, we obtain (10).

By the argument of Lemma 3, we obtain the following result.

Theorem 2. Let $T=\inf \left\{t \mid W_{t}=0\right\}$, where $W_{0}$ is distributed according to the stationary distribution. Then the mean length of the busy period is given by

$$
\mathbb{E} T=\frac{\mathbb{E} W}{(1-p) \varphi^{\prime}(0)} .
$$

Remark 2. Note that for the $M / G / 1$ queue with server breakdowns and multiple vacations, if $B_{i}$ is distributed as a service time, then $T_{n}$ is the n-order busy period which is defined as the time period which starts when a vacation is completed and there are $n$ customers in the system and ends at the next departure epoch when the system is empty.

\section{The Transient Distribution}

In this section, we focus on analyzing the transient distribution of $W_{t}$ in terms of Laplace-Stieltjes transform, for some $t>0$, conditional on $W_{0}=x$.

Theorem 3. Let $T$ be exponentially distributed with mean $1 / \gamma$, independently of $Y, \tau_{n}$ and $\sigma_{n}, n \geq 1$. For $\theta>0$ and $x \geq 0$,

$$
\begin{aligned}
\mathbb{E}_{x} e^{-\theta W_{T}}= & \frac{\gamma}{\varphi(\theta)-\gamma} e^{-\varphi^{-1}(\gamma) x} \\
& \times\left\{p \frac{\mathbb{E} e^{-\theta W^{-}}-\mathbb{E} e^{-\theta W^{+}}}{\mathbb{E} e^{-\varphi^{-1}(\gamma) W^{-}}-\mathbb{E} e^{-\varphi^{-1}(\gamma) W^{+}}}+(1-p) \frac{1-\mathbb{E} e^{-\theta \eta_{1}}}{1-\mathbb{E} e^{-\varphi^{-1}(\gamma) \eta_{1}}}-e^{-\left[\theta-\varphi^{-1}(\gamma)\right] x}\right\} .
\end{aligned}
$$

Proof of Theorem 3. By Lemma 1, we have

$$
\begin{aligned}
0= & \mathbb{E} M_{T}=\varphi(\theta) \int_{0}^{\infty} \int_{0}^{t} \gamma e^{-\gamma t} e^{-\theta W_{s}} d s d t+e^{-\theta x}-\mathbb{E}_{x} e^{-\theta W_{T}} \\
& -\mathbb{E} \sum_{k=1}^{N_{T}^{R}}\left[e^{-\theta\left(W_{\tau_{k}}-\tau_{k}\right)}-e^{-\theta W_{\tau_{k}}}\right]-\mathbb{E} \sum_{k=1}^{N_{T}^{V}}\left(1-e^{-\theta \eta_{k}}\right) \\
= & \frac{\varphi(\theta)}{\gamma} \mathbb{E}_{x} e^{-\theta W_{T}}+e^{-\theta x}-\mathbb{E}_{x} e^{-\theta W_{T}}-\mathbb{E} N_{T}^{R}\left[\mathbb{E} e^{-\theta W^{-}}-\mathbb{E} e^{-\theta W^{+}}\right] \\
& -\mathbb{E} N_{T}^{V}\left(1-\mathbb{E} e^{-\theta \eta_{1}}\right) .
\end{aligned}
$$

So that

$$
\mathbb{E}_{x} e^{-\theta W_{T}}=\frac{\gamma}{\varphi(\theta)-\gamma}\left\{\mathbb{E} N_{T}^{R}\left[\mathbb{E} e^{-\theta W^{-}}-\mathbb{E} e^{-\theta W^{+}}\right]+\mathbb{E} N_{T}^{V}\left(1-\mathbb{E} e^{-\theta \eta_{1}}\right)-e^{-\theta x}\right\}
$$


Note that when $\mathbb{E} Y_{1}<0, \varphi(\theta)$ is increasing on $[0, \infty)$. Therefore, the inverse of $\varphi(\theta)$ is well defined on $[0, \infty)$. Hence, the equation $\varphi(\theta)=\gamma$ has exactly one $\operatorname{root} \theta=\varphi^{-1}(\gamma)$ on $[0, \infty)$. Finally, using the fact that the root of the denominator should be a root of the numerator as well, we can obtain (11).

Next, we derive explicitly the Laplace transform corresponding to the correlation of the workload process:

$$
c(t)=\frac{\operatorname{Cov}\left(W_{0}, W_{t}\right)}{\sqrt{\operatorname{Var} W_{0} \cdot \operatorname{Var} W_{t}}}=\frac{\mathbb{E}\left(W_{0} W_{t}\right)-\left(\mathbb{E} W_{0}\right)^{2}}{\operatorname{Var} W_{0}}=\frac{\mathbb{E}\left(W_{0} W_{t}\right)-\omega^{2}}{v} .
$$

Here, we assume the system is in steady-state at time 0 .

Theorem 4. For $\gamma>0$ and $\omega, v$ as in Corollary 1,

$$
\begin{aligned}
\int_{0}^{\infty} c(t) e^{-\gamma t} d t= & \frac{1}{\gamma}-\frac{\omega \varphi^{\prime}(0)}{v \gamma^{2}}+\frac{\mathbb{E}\left(W e^{-\varphi^{-1}(\gamma) W}\right)}{v \gamma} \\
& \times\left\{\frac{p \mathbb{E} \xi_{1}}{\mathbb{E} e^{-\varphi^{-1}(\gamma) W^{-}}-\mathbb{E} e^{-\varphi^{-1}(\gamma) W^{+}}}+\frac{(1-p) \mathbb{E} \eta_{1}}{1-\mathbb{E} e^{-\varphi^{-1}(\gamma) \eta_{1}}}\right\},
\end{aligned}
$$

where

$$
\begin{aligned}
\mathbb{E}\left(W e^{-\varphi^{-1}(\gamma) W}\right)= & {\left[\frac{\varphi^{\prime}(0)}{\varphi(\gamma)}-\frac{\gamma \varphi^{\prime}(0) \varphi^{\prime}(\gamma)}{\varphi^{2}(\gamma)}\right]\left[p \frac{\mathbb{E} e^{-\gamma W^{-}}-\mathbb{E} e^{-\gamma W^{+}}}{\theta \mathbb{E} \xi_{1}}+(1-p) \frac{1-\mathbb{E} e^{-\gamma \eta_{1}}}{\gamma \mathbb{E} \eta_{1}}\right] } \\
& +\frac{\varphi^{\prime}(0)}{\varphi(\gamma)} \frac{p}{\mathbb{E} \xi_{1}}\left[\mathbb{E}\left(W^{+} e^{-\gamma W^{+}}\right)-\mathbb{E}\left(W^{-} e^{-\gamma W^{-}}\right)-\frac{\mathbb{E} e^{-\gamma W^{-}}-\mathbb{E} e^{-\gamma W^{+}}}{\gamma}\right] \\
& +\frac{\varphi^{\prime}(0)}{\varphi(\gamma)} \frac{1-p}{\mathbb{E} \eta_{1}}\left[\mathbb{E}\left(\eta_{1} e^{-\gamma \eta_{1}}\right)-\frac{1-\mathbb{E} e^{-\gamma \eta_{1}}}{\gamma}\right] .
\end{aligned}
$$

Proof of Theorem 4. Let $T$ be exponentially distributed with mean $1 / \gamma$. From (11), we get

$$
\int_{0}^{\infty} \gamma e^{-\gamma t} \mathbb{E}_{x} W_{t} d t=-\frac{\varphi^{\prime}(0)}{\gamma}+e^{-\varphi^{-1}(\gamma) x}\left[\frac{p \mathbb{E} \xi_{1}}{\mathbb{E} e^{-\varphi^{-1}(\gamma) W^{-}}-\mathbb{E} e^{-\varphi^{-1}(\gamma) W^{+}}}+\frac{(1-p) \mathbb{E} \eta_{1}}{1-\mathbb{E} e^{-\varphi^{-1}(\gamma) \eta_{1}}}\right]+x .
$$

Straightforward calculus yields

$$
\begin{aligned}
\int_{0}^{\infty} c(t) e^{-\gamma t} d t & =\frac{1}{v} \int_{0}^{\infty}\left[\mathbb{E}\left(W_{0} W_{t}\right)-\omega^{2}\right] e^{-\gamma t} d t \\
& =\frac{1}{v} \int_{0}^{\infty} \int_{0}^{\infty} x \mathbb{E}_{x} W_{t} e^{-\gamma t} d \mathbb{P}\left(W_{0} \leq x\right) d t-\frac{\omega^{2}}{v \gamma}
\end{aligned}
$$

Substituting (12) into (13), we obtain

$$
\begin{aligned}
& \int_{0}^{\infty} c(t) e^{-\gamma t} d t=-\frac{\omega^{2}}{v \gamma}+ \\
& \int_{0}^{\infty} \frac{x}{v \gamma}\left\{-\frac{\varphi^{\prime}(0)}{\gamma}+e^{-\varphi^{-1}(\gamma) x}\left[\frac{p \mathbb{E} \xi_{1}}{\mathbb{E} e^{-\varphi^{-1}(\gamma) W^{-}}-\mathbb{E} e^{-\varphi^{-1}(\gamma) W^{+}}}+\frac{(1-p) \mathbb{E} \eta_{1}}{1-\mathbb{E} e^{-\varphi^{-1}(\gamma) \eta_{1}}}\right]+x\right\} d \mathbb{P}\left(W_{0} \leq x\right) \\
& =\frac{1}{\gamma}-\frac{\omega \varphi^{\prime}(0)}{v \gamma^{2}}+\frac{\mathbb{E}\left(W e^{-\varphi^{-1}(\gamma) W}\right)}{v \gamma}\left\{\frac{p \mathbb{E} \xi_{1}}{\mathbb{E} e^{-\varphi^{-1}(\gamma) W^{-}}-\mathbb{E} e^{-\varphi^{-1}(\gamma) W^{+}}}+\frac{(1-p) \mathbb{E} \eta_{1}}{1-\mathbb{E} e^{-\varphi^{-1}(\gamma) \eta_{1}}}\right\},
\end{aligned}
$$

where $\mathbb{E}\left(W e^{-\varphi^{-1}(\gamma) W}\right)$ can be obtained by differentiating (5). This completes the proof. 


\section{Stochastic Decompositions}

We now identify the stochastic decomposition properties of the limiting distribution of the workload process for our fluid queueing system with Lévy input. Stochastic decomposition properties have been investigated in many queueing systems with vacations (see [10]). The well-known classical stochastic decomposition property shows that the random variable of stationary system size at an arbitrary point can be represented as the sum of two independent random variables, one of which is the steady-state system size of the corresponding standard queueing system without server vacations and the other random variable is the queue size given that the server is on vacations. It may depend on the meaning of vacations in specific cases (see Fuhrmann and Cooper [26] and Doshi [27]). In addition, stochastic decomposition properties have also been held for Lévy-driven queues with interruptions as well as càdlàg processes with certain secondary jump inputs (see Kella and Whitt [11] and Ivanovs and Kella [28]). It's worth mentioning that, recently, Boxma and Kella [17] generalize the well-known workload process decomposition results for Lévy queues with secondary jump inputs and queues with server vacations or service interruptions. In particular, in the context of our fluid queueing system, we have the following decomposition results.

Lemma 4. There are two random variables $U$ and $V$ such that

$$
\begin{gathered}
\frac{\mathbb{E} e^{-\theta W^{-}}-\mathbb{E} e^{-\theta W^{+}}}{\theta \mathbb{E} \xi_{1}}=\mathbb{E} e^{-\theta U}, \\
\frac{1-\mathbb{E} e^{-\theta \eta_{1}}}{\theta \mathbb{E} \eta_{1}}=\mathbb{E} e^{-\theta V} .
\end{gathered}
$$

Proof of Lemma 4. The left hand side of (14) is the Laplace transformation of the function

$$
f(x)=\frac{\mathbb{P}\left(W^{+}>x\right)-\mathbb{P}\left(W^{-}>x\right)}{\mathbb{E} \xi_{1}},
$$

which is a bona fide probability density function (note that $f(x)$ is the stationary residual life distribution of $\xi_{1}$ ).

The left hand side of (15) is the Laplace transformation of the function

$$
g(x)=\frac{\mathbb{P}\left(\eta_{1}>x\right)}{\mathbb{E} \eta_{1}}
$$

which is a bona fide probability density function (stationary residual life density of $\eta_{1}$ ).

Applying Lemma 4, we can get the following theorem to characterize the stochastic decomposition property.

Theorem 5. Under the conditions of Theorem 1, the distribution of $W$ is the convolution of two distributions one of which is the distribution of $R$ in Section 2 and the other is the mixture of the distribution of $U$ with probability $p$ and the stationary residual life distribution of $\eta_{1}$ with probability $1-p$.

Furthermore, under some conditions, we have another stochastic decomposition result.

Lemma 5. Under the conditions of Theorem 1 , if positive random variables $\xi_{n}, n \geq 1$ are independent of $W_{0}$, $Y_{\tau_{n}}$ and $\sum_{i=1}^{N_{\tau_{n}}^{V}} \eta_{i}$, then we have

$$
\mathbb{E} e^{-\theta W}=\frac{\theta \varphi^{\prime}(0)}{\varphi(\theta}\left[p \frac{1-\mathbb{E} e^{-\theta \xi_{1}}}{\theta \mathbb{E} \xi_{1}} \mathbb{E} e^{-\theta W^{-}}+(1-p) \frac{1-\mathbb{E} e^{-\theta \eta_{1}}}{\theta \mathbb{E} \eta_{1}}\right] .
$$


Proof of Lemma 5. Since $\xi_{k}$ is independent of $W_{\tau_{k}}-\xi_{k}=W_{0}+Y_{\tau_{n}}+\sum_{i=1}^{k-1} \xi_{i}+\sum_{i=1}^{N_{\tau_{k}}^{V}} \eta_{i}$,

$$
\begin{aligned}
\frac{\mathbb{E} e^{-\theta\left(W_{\tau_{1}}-\xi_{1}\right)}-\mathbb{E} e^{-\theta W_{\tau_{1}}}}{\theta \mathbb{E} \xi_{1}} & =\frac{\mathbb{E} e^{-\theta\left(W_{\tau_{1}}-\xi_{1}\right)}\left(1-e^{-\theta \xi_{1}}\right)}{\theta \mathbb{E} \xi_{1}} \\
& =\mathbb{E} e^{-\theta\left(W_{\tau_{1}}-\xi_{1}\right)} \frac{1-\mathbb{E} e^{-\theta \xi_{1}}}{\theta \mathbb{E} \xi_{1}} \\
& =\mathbb{E} e^{-\theta W^{-}} \frac{1-\mathbb{E} e^{-\theta \xi_{1}}}{\theta \mathbb{E} \xi_{1}} .
\end{aligned}
$$

Substituting (17) into (5) yields (16).

From Lemma 5, we obtain the following theorem which provides another stochastic decomposition.

Theorem 6. If the assumptions of Lemma 5 are satisfied, then the distribution of $U$ is the convolution of the distribution of $W^{-}$and the stationary residual life distribution of $\xi_{1}$.

\section{Conclusions}

In this paper, we have investigated a single-server fluid queue with Lévy input. The server may take multiple vacations and is subject to breakdowns and repairs. A breakdown at the server is represented by the arrival of a failure. The behavior of this stochastic system is described by a generalized Lévy process. Applying the Kella-Whitt martingale technology, we study the stochastic dynamics of the system and realize an extensive analysis of the system from the transient virtual waiting time process to the steady-state distribution of the waiting time process. Furthermore, we give a detailed analysis of the system busy period. Finally, we establish the stochastic decomposition on the limiting distribution of the workload process. Our results extend and unify previous results for $M / G / 1$ queue with server breakdowns and vacations and Lévy-driven queues with server vacations. Fluid queues with Lévy input are very good mathematical models for cellular mobile communication networks, data communication networks, public switched telephone network, local area networks under the protocols of random multiple access, computer systems for competing to gain service from a central processor unit, etc. Future work will consider the optimal control of stochastic service systems with Lévy input and their applications to computer communication networks.

Author Contributions: Formal analysis, Y.P.; Methodology, J.W. All authors have read and agree to the published version of the manuscript.

Funding: This research is supported by Provincial Natural Science Foundation of Hunan under Grant 2019JJ50677, 2020JJ4760 and the Program of Hehua Excellent Young Talents of Changsha Normal University.

Acknowledgments: The authors would like to thank four anonymous referees for their valuable comments and suggestions which have definitely helped to improve the presentation of this paper.

Conflicts of Interest: The authors declare no conflict of interest.

\section{References}

1. Bertoin, J. Lévy Processes; Cambridge University Press: Cambridge, UK, 1998.

2. Sato, K. Léoy Processes and Infinitely Divisible Distributions; Cambridge University Press: Cambridge, UK, 1999.

3. Applebaum, D. Léry Processes and Stochastic Calculus; Cambridge University Press: Cambridge, UK, 2004.

4. Kyprianou, A. Introductory Lectures on Fluctuations of Lévy Processes with Applications; Springer Press: Berlin, Germany, 2006.

5. Gray, W.J.; Wang, P.P.; Scott, M. A vacation queueing model with service breakdowns. Appl. Math. Model. 2000, 24, 391-400. [CrossRef]

6. Ke, J.C. Optimal strategy policy in batch arrival queue with server breakdowns and multiple vacations. Math. Method. Oper. Res. 2003, 58, 41-56. [CrossRef] 
7. Jain, M.; Jain, A. Working vacations queueing model with multiple types. Appl. Math. Model. 2010, 34, 1-13. [CrossRef]

8. Wu, J.; Yin, X. An M/G/1 retrial G-queue with non-exhaustive random vacations and unreliable server. Comput. Math. Appl. 2011, 62, 2314-2329. [CrossRef]

9. Yang, D.Y.; Wu, C.H. Cost-minimization analysis of a working vacation queue with N-policy and server breakdowns. Comput. Ind. Eng. 2015, 82, 151-158. [CrossRef]

10. Tian, N.; Zhang, Z.G. Vacation Queueing Models-Theory and Applications; Springer: New York, NY, USA, 2006.

11. Kella, O.; Whitt, W. Queues with server vacations and Lévy processes with secondary jump input. Ann. Appl. Probab. 1991, 1, 104-117. [CrossRef]

12. Lieshout, P.; Mandjes, M. Asymptotic analysis of Lévy-driven tandem queues. Queueing Syst. 2008, 60, 203-226. [CrossRef]

13. Boxma, O.; Kella, O. On a generic class of Lévy-driven vacation models. Probab. Eng. Inf. Sci. 2010, $24,1-12$. [CrossRef]

14. Debicki, K.; Mandjes, M. Lévy-driven queues. Surv. Oper. Res. Manag. Sci. 2012, 17, 15-37. [CrossRef]

15. Dębicki, K.; Mandjes, M.; Sierpinska-Tulacz, I. Transient analysis of Lévy-driven tandem queues. Stat. Probab. Lett. 2013, 83, 1776-1781. [CrossRef]

16. Palmowski, Z.; Vlasiou, M.; Zwart, B. A Lévy input fluid queue with input and workload regulation. Queueing Syst. 2014, 76, 21-36. [CrossRef]

17. Boxma, O.; Kella, O. Decomposition results for stochastic storage processes and queues with alternating Lévy inputs. Queueing Syst. 2014, 77, 97-112. [CrossRef]

18. Ivanovs, J.; Mandjes, M. Transient analysis of Lévy-driven tandem queues. Stat. Probab. Lett. 2015, 107, 341-347. [CrossRef]

19. Koops, D.T.; Boxma, O.J.; Mandjes, M. A tandem fluid network with Lévy input in heavy traffic. Queueing Syst. 2016, 84, 355-379. [CrossRef]

20. Debicki, K.; Liu, P.; Mandjes, M.; Sierpinska-Tulacz, I. Lévy-driven GPS queues with heavy-tailed input. Queueing Syst. 2017, 85, 249-267. [CrossRef]

21. Dai, W. A unified system of FB-SDEs with Lévy jumps and double completely-S skew reflections. Commun. Math. Sci. 2018, 16, 659-704. [CrossRef]

22. Ravner, L.; Boxma, O.; Mandjes, M. Estimating the input of a Lévy-driven queue by Poisson sampling of the workload process. Bernoulli 2019, 25, 3734-3761. [CrossRef]

23. Berkelmansa, W.; Cichockaa, A.; Mandjes, M. The correlation function of a queue with Lévy and Markov additive input. Stoch. Proc. Appl. 2020, 130, 1713-1734. [CrossRef]

24. Kella, O.; Whitt, W. Useful martingales for stochastic processes with Lévy input. J. Appl. Probab. 1992, 29, 396-403. [CrossRef]

25. Chung, K.L. A Course in Probability Theory, 2nd ed.; Academic Press: New York, NY, USA; London, UK, 1974.

26. Fuhrmann, S.W.; Cooper, R.B. Stochastic decomposition in the M/G/1 queue with generalized vacations. Oper. Res. 1985, 33, 1117-1129. [CrossRef]

27. Doshi, B. Conditional and unconditional distributions for $M / G / 1$ type queues with server vacations. Queueing Syst. 1990, 7, 229-252. [CrossRef]

28. Ivanovs, J.; Kella, O. Another look into decomposition results. Queueing Syst. 2013, 75, 19-28. [CrossRef]

(C) 2020 by the authors. Licensee MDPI, Basel, Switzerland. This article is an open access article distributed under the terms and conditions of the Creative Commons Attribution (CC BY) license (http:/ / creativecommons.org/licenses/by/4.0/). 